\title{
Laying the foundations for quality improvement within an NHS trust: A chief registrar's journey
}

\author{
Author: Christopher Huntley*
}

\section{Introduction}

The benefit of quality improvement (QI) is acknowledged nationally across the NHS, royal colleges, regulatory bodies and in under- and postgraduate curricula. However, disparity remains between idealistic QI practice and local trust realities. This is a summary of a chief registrar's experience when attempting to lay the foundations for a QI service at the Royal Wolverhampton NHS Trust (RWT).

\section{Methods}

A QI strategy for the 2018-19 academic year was developed and delivered by the RCP chief registrar with support from the divisional medical director and key stakeholders. This focused on four key elements: QI education; QI governance; QI support; and sharing learning from QI work. Doctors in training (DiT) regularly completed 10-item Likert scales and qualitative surveys to assess the impact of our strategy.

\section{Results and discussion}

\section{QI education and support}

A new four-session QI workshop programme with supplementary drop-in sessions has been developed and provided education and support. To date, $127 \mathrm{DiT}$ are enrolled, contributing to a minimum of 20 QI projects. The programme has been well received with improvements demonstrated in DiT confidence in leading QI work, self-rating of medical leadership qualities and a sense of empowerment (Fig 1). The first cohort of supervisors, nurses and governance managers have now been enrolled to this programme, to address supervision barriers identified.

\section{Governance and learning from QI}

Creating a divisional QI working group has enabled a review of current governance processes and we are now embedding QI within this, alongside developing a QI database. The first RWT QI conference is planned for May 2019, showcasing the ongoing QI work, aiming to improve wider engagement with QI and gather support for continuing projects. From April 2019, a trust QI team has been established which will build on these foundations. This has led to an improvement in the perceived QI culture at RWT (Fig 1).

Author: New Cross Hospital, Wolverhampton ${ }^{*} \mathrm{RCP}$ chief registrar

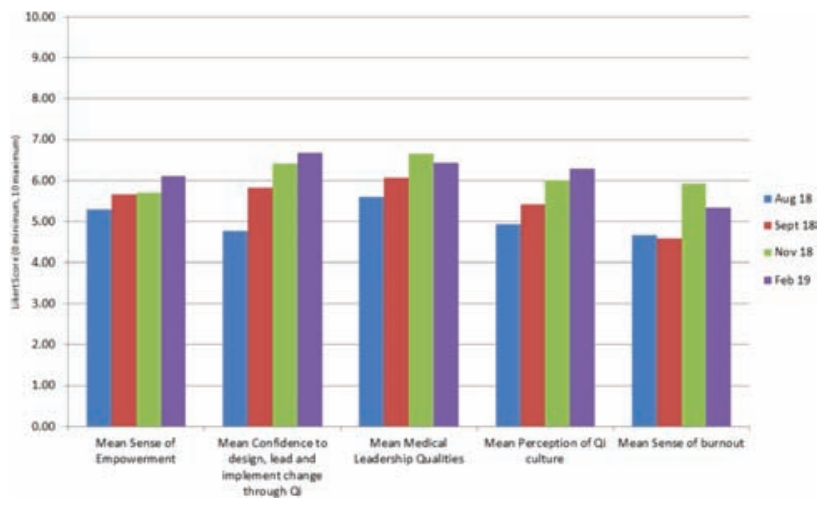

Fig 1. Doctors in training mean Likert scale by month

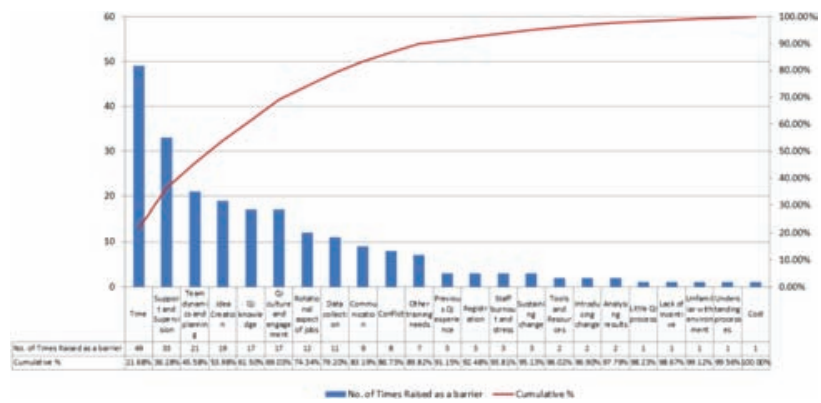

Fig 2. Pareto graph of perceived barriers to quality improvement work

Through this journey, identifying and addressing challenges and barriers to QI (Fig 2) has been key to directing future developments. Focus now centres on a multidisciplinary approach to QI and 'upscaling' learning from changes introduced.

\section{Conclusion}

If QI is to succeed and avoid previous mistakes of traditional clinical audit, engagement is required trust-wide, from groundstaff to executive management. Understanding the barriers individuals undertaking QI work face and overcoming these is critical in establishing a successful QI infrastructure. We highlight and address fundamental barriers in QI education, support and engagement with QI work, through our QI strategy. We have laid the foundations from which the newly formed QI team can develop within RWT over the coming years. 